\title{
Hyperoxia may be related to delayed cerebral ischemia and poor outcome after subarachnoid haemorrhage
}

\author{
Sang-Beom Jeon, ${ }^{1,2,3}$ H Alex Choi, ${ }^{2,3}$ Neeraj Badjatia, ${ }^{3,4}{ }^{3}$ Michael Schmidt, ${ }^{3}$ \\ Hector Lantigua, ${ }^{3}$ Jan Claassen, ${ }^{3}$ E Sander Connolly, ${ }^{5}$ Stephan A Mayer, ${ }^{3}$ \\ Kiwon Lee Le, $^{2,3}$
}

\begin{abstract}
- Additional material is published online only. To view please visit the journal online (http://dx.doi.org/10.1136/ jnnp-2013-307314).

For numbered affiliations see end of article.
\end{abstract}

Correspondence to Dr Kiwon Lee, Departments of Neurology and Neurosurgery, The University of Texas Medical School at Houston, 6431 Fannin St., MSB 7.152, Houston, TX 77030, USA; Kiwon.Lee@uth.tmc.edu

Received 25 November 2013 Revised 17 April 2014 Accepted 4 May 2014 Published Online First 23 May 2014

\section{SLinked}

http://dx.doi.org/10.1136/ jnnp-2014-308326

\section{CrossMark}

To cite: Jeon S-B, Choi HA, Badjatia N, et al. J Neurol Neurosurg Psychiatry 2014;85:1301-1307.

\section{ABSTRACT}

Objective To determine the association between exposure to hyperoxia and the risk of delayed cerebral ischaemia (DCI) after subarachnoid haemorrhage (SAH). Methods We analysed data from a single centre, prospective, observational cohort database. Patient inclusion criteria were age $\geq 18$ years, aneurysmal $\mathrm{SAH}$, endotracheal intubation with mechanical ventilation, and arterial partial pressure of oxygen $\left(\mathrm{PaO}_{2}\right)$ measurements. Hyperoxia was defined as the highest quartile of an area under the curve of $\mathrm{PaO}_{2}$, until the development of $\mathrm{DCl}$ $\left(\mathrm{PaO}_{2} \geq 173 \mathrm{~mm} \mathrm{Hg}\right)$. Poor outcome was defined as modified Rankin Scale 4-6 at 3 months after SAH.

Results of 252 patients, there were no differences in baseline characteristics between the hyperoxia and control group. Ninety-seven (38.5\%) patients developed $\mathrm{DCl}$. The hyperoxia group had a higher incidence of $\mathrm{DCl}$ $(p<0.001)$ and poor outcome $(p=0.087)$. After adjusting for modified Fisher scale, rebleeding, global cerebral oedema, intracranial pressure crisis, pneumonia and sepsis, hyperoxia was independently associated with $D C l$ (OR, 3.16; $95 \% \mathrm{Cl} 1.69$ to $5.92 ; \mathrm{p}<0.001)$. After adjusting for age, Hunt-Hess grade, aneurysm size, Acute Physiology and Chronic Health Evaluation II score, rebleeding, pneumonia and sepsis, hyperoxia was independently associated with poor outcome (OR, 2.30; $95 \% \mathrm{Cl} 1.03$ to $5.12 ; \mathrm{p}=0.042$ ).

Conclusions In SAH patients, exposure to hyperoxia was associated with $\mathrm{DCl}$. Our findings suggest that exposure to excess oxygen after $\mathrm{SAH}$ may represent a modifiable factor for morbidity and mortality in this population.

\section{INTRODUCTION}

Supplemental oxygen is commonly administered to patients after subarachnoid haemorrhage (SAH) during the prehospital, emergency and critical care. ${ }^{1}$ During mechanical ventilation, hyperoxia is not uncommon in the emergency room and intensive care unit during the management of SAH. Recent studies in patients after cardiac arrest and traumatic brain injury have suggested that hyperoxia is associated with worse outcomes. ${ }^{2} 3$ Contrarily, others have suggested that hyperoxia may be of therapeutic benefit for patients with traumatic brain injury and ischaemic stroke. ${ }^{4-7}$ The effect of hyperoxia on outcomes in patients with SAH has not been studied.
At supra-physiologic levels, oxygen may have toxic effects on the human brain through production of superoxide anion, hydrogen peroxide and hydroxyl radical. ${ }^{8}$ The overproduction of these reactive oxygen species results in oxidative stress, a deleterious process which may lead to neuronal inflammation and necrosis as well as reduction of cerebral blood flow. ${ }^{9}{ }^{10}$ After SAH, these processes may contribute to the development of delayed cerebral ischaemia (DCI).

We hypothesised that hyperoxia may increase the risk of DCI after SAH. We aimed to investigate the association between exposure to hyperoxia and the incidence of DCI and poor 3-month outcome.

\section{PATIENTS AND METHODS}

Patients

Columbia University SAH Outcomes Database Project is a single centre, prospective observational cohort study. Data fields include demographics, clinical, radiological and outcome data for all adult spontaneous SAH patients. All data are prospectively collected by a dedicated investigator and are adjudicated by the study team in weekly meetings. ${ }^{11} 12$ This retrospective analysis considered all SAH patients collected in our registry between August 1996 and April 2011. We included patients who (1) were 18 years of age or older; (2) had aneurysmal SAH confirmed by brain CT with angiography and/or CT angiography; (3) were endotracheally intubated with ventilators within 3 days after onset (excluding patients intubated solely for surgical interventions); (4) underwent arterial partial pressure of oxygen $\left(\mathrm{PaO}_{2}\right)$ measurements $\geq 3$ times (to be able to calculate area under the curve) within 14 days, including twice or more within 3 days. We excluded patients who expired within 14 days after symptom onset because (1) early death would lead to less chance of DCI; (2) it was uncertain that the cause of early death was related to DCI; (3) severe hypoxia could precede death.

This study was approved by the institutional review board of our centre. Informed consents for this study were obtained from patients or their family members.

Clinical, radiological and laboratory assessment We obtained demographics (age, sex and ethnicity), risk factors or underlying comorbidities 
(hypertension, smoking, alcohol consumption, previous ischaemic or haemorrhagic stroke, cardiac disease, such as myocardial infarction and arrhythmia, and pulmonary disease, such as chronic obstructive pulmonary disease), clinical, radiological and laboratory findings on initial assessments (Glasgow coma scale (GCS), Hunt-Hess grade, modified Fisher scale, ${ }^{13}$ Hijdra scale, ${ }^{13}$ global cerebral oedema on initial CT, ${ }^{14}$ APACHE II (Acute Physiology and Chronic Health Evaluation II) score, $\mathrm{PaO}_{2}$, and arterial partial pressure of carbon dioxide), and acute complications of SAH on follow-up assessments (rebleeding, ${ }^{15}$ global cerebral oedema on follow-up CT, hydrocephalus, intracranial pressure crisis, meningitis, pneumonia or pulmonary oedema, and sepsis), DCI, and modified Rankin Scale (mRS). ${ }^{15}$

\section{Definitions of hyperoxia and other variables}

Oxygen burden was defined as the area under the curve of $\mathrm{PaO}_{2}$ divided by the observation period since the onset of SAH. The main objectives of this study were to investigate the effects of hyperoxia on DCI, which mostly occurs within the first 2 weeks. For this purpose, oxygen burden was calculated until the time point when each patient developed DCI. For patients who did not develop DCI, oxygen burden until postbleed day 6 was adopted for comparison. Six days were the median day from symptom onset to DCI in our study population. We further defined hyperoxia as the uppermost quartile of oxygen burden. The median oxygen burden was $146.5 \mathrm{~mm} \mathrm{Hg}$ (IQR, 123$173 \mathrm{~mm} \mathrm{Hg}$ ) (figure 1). Thus, oxygen burden $\geq 173 \mathrm{~mm} \mathrm{Hg}$ was considered as hyperoxia, as aforementioned. We chose these cutoff points, because (1) we investigated the 'excess' oxygen's effects and (2) there is no consensus on a specific cutoff point of hyperoxia in a human SAH population.

DCI was defined as either a symptomatic vasospasm (ie, clinical deterioration, such as a new focal deficit and/or a decrease of the level of consciousness, in the presence of vasospasm determined by conventional angiography or CT angiography) or development of new cerebral infarction which is not explained by other causes such as hydrocephalus, seizures, intracranial pressure crisis and infection. ${ }^{16}$ This definition of DCI has been demonstrated to have a better predictive value for poor clinical outcomes than symptomatic, angiographic, or transcranial Doppler vasospasm. ${ }^{16} \mathrm{mRS}$ was obtained by in-person interview

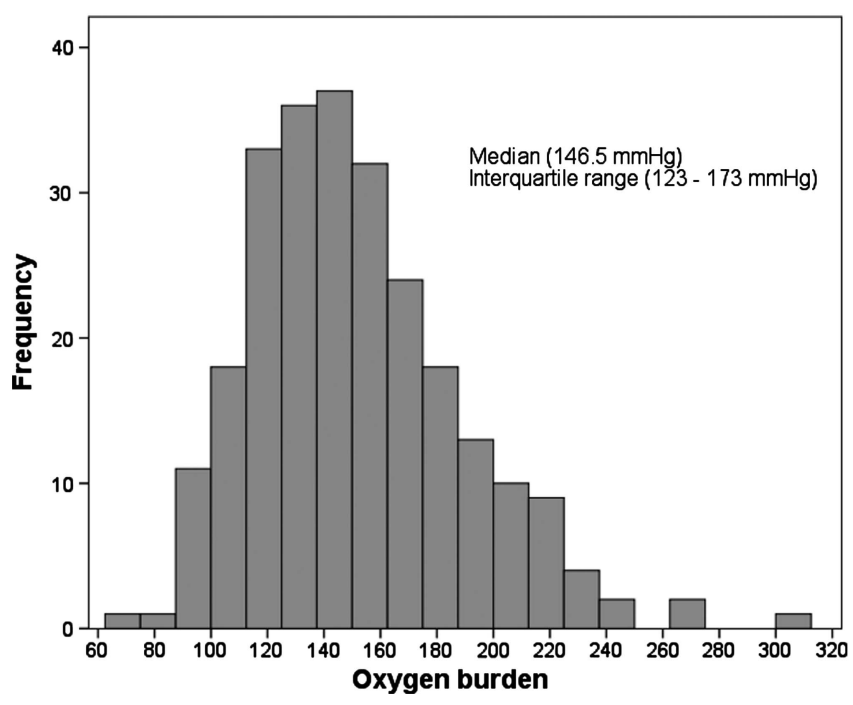

Figure 1 Distributions of oxygen burden. or phone interview either with the patient or with a surrogate if the patient was unable to participate. Poor outcome was defined as mRS $4-6$ at 3 months after the onset of SAH. ${ }^{16}$ We recorded the day of aneurysmal rupture as bleed day 0 .

\section{Clinical managements}

The management of these patients followed guidelines set forth by the American Heart Association and has been described in detail previously. ${ }^{15}$ Endotracheal intubation was performed and mechanical ventilator was applied to the patients whose respiratory drive or reflexes were abnormal. All patients underwent neurological examinations each day. Patients underwent conventional angiography on admission, and between postbleed days 4 and 8 if clinically indicated. Transcranial Doppler was performed daily, and CT angiography was performed for clinical purposes. Patients who developed DCI were treated with hypertensive hypervolemic therapy. Endovascular treatment, including intra-arterial verapamil hydrochloride injection and/or angioplasty, was attempted on patients who developed DCI and did not respond to medical treatment. We did not increase fraction of inspired oxygen as a modality of prevention or treatment of DCI. ${ }^{15}$

\section{Data analysis}

We compared demographics, risk factors and comorbidities, findings on initial and follow-up assessments, and the exposure to hyperoxia with the development of DCI, and also with poor outcome. For univariate analysis, we used Pearson's $\chi^{2}$ test with the exact method, Student $t$ test and the Mann-Whitney U test as appropriate. Multiple logistic regression analysis was performed after examining collinearity to estimate the independent contributions of variables to DCI and poor outcome. Variables with a $\mathrm{p}$ value $<0.1$ by univariate analysis were included in the multivariable model as candidate variables, and then removed by a backward stepwise selection procedure. We further performed all analysis using a forward selection procedure. A twotailed $p$ value of $<0.05$ was considered to indicate a significant difference in all statistical analysis. SPSS for Windows V.13.0 (SPSS, Chicago, Illinois) was used for all statistical analyses.

\section{RESULTS}

During the study period, 1140 patients with aneurysmal SAH were identified. Of those, 888 patients did not meet inclusion criteria and were excluded. Thus, the remaining 252 patients were included for the final analysis. Age was the only baseline characteristic that was different between 252 included and 888 excluded subjects, included patients being older (mean $\pm S D$, $56.7 \pm 13.9$ years vs $53.7 \pm 14.7$ years; $\mathrm{p}=0.005$ ). SAH severity was more severe in included patients compared with excluded patients: GCS (median (IQR), $8 \quad(6-10)$ vs 15 (12-15); $\mathrm{p}<0.001$ ); Hunt-Hess grade (grade $1,4 \%$ vs $29 \%$; grade $2,4 \%$ vs $22 \%$; grade $3,25 \%$ vs $30 \%$; grade $4,43 \%$ vs $9 \%$; grade 5 , $24 \%$ vs $10 \%$; $<0.001$ ); modified Fisher scale (group 0, 0\% vs $4 \%$; group $1,10 \%$ vs $33 \%$; group 2 , $11 \%$ vs $9 \%$; group 3 , $41 \%$ vs $37 \%$; group $4,38 \%$ vs $17 \%$; $<0.001)$; and APACH II score (mean $\pm S D, 17.7 \pm 6.7$ vs $10.6 \pm 7.2 ; p<0.001$ ) (see online supplementary table $\mathrm{S} 1)$.

Of the 252 included patients, 179 (71\%) were women. A mean age was $56.7 \pm 13.9$ years. The median time from symptom onset to the initial $\mathrm{PaO}_{2}$ was $16.4 \mathrm{~h}$ (IQR, 10.3-25.9 h). The median frequency of $\mathrm{PaO}_{2}$ measurements, within 14 days after symptom onset, was 23.0 (IQR, 15-31). Initial Hunt-Hess grade ranged from grade 1 to grade 5 , with high grades predominant (table 1 ). 
Table 1 Patient characteristics

\begin{tabular}{|c|c|c|c|c|}
\hline & \multicolumn{2}{|c|}{ Delayed cerebral ischaemia } & \multicolumn{2}{|c|}{ Poor outcome } \\
\hline & No $(n=155)$ & Yes $(n=97)$ & No $(n=94)$ & Yes $(n=108)$ \\
\hline \multicolumn{5}{|l|}{ Demographics } \\
\hline Age, years & $57.1 \pm 14.1$ & $56.0 \pm 13.5$ & $53.1 \pm 12.0$ & $60.5 \pm 15.6$ \\
\hline Gender, male & $47(30.3)$ & $26(26.8)$ & $34(36.2)$ & $28(25.9)$ \\
\hline Ethnicity, white & $68(43.9)$ & $41(42.3)$ & $45(47.9)$ & $51(47.2)$ \\
\hline \multicolumn{5}{|l|}{ Risk factors and comorbidities } \\
\hline Hypertension & $85(54.8)$ & $46(47.4)$ & $46(48.9)$ & $56(51.9)$ \\
\hline Smoking & $61(39.4)$ & $34(35.1)$ & $40(42.6)$ & $29(26.9)$ \\
\hline Alcohol & $14(9.0)$ & $11(11.3)$ & $12(12.8)$ & $6(5.6)$ \\
\hline Stroke & $12(7.7)$ & $4(4.1)$ & $3(3.2)$ & $11(10.2)$ \\
\hline Cardiac disease & $16(10.3)$ & $8(8.2)$ & $4(4.3)$ & $17(15.7)$ \\
\hline Pulmonary disease & $10(6.5)$ & $4(4.1)$ & $6(6.4)$ & $7(6.5)$ \\
\hline \multicolumn{5}{|l|}{ Initial assessment } \\
\hline GCS & $8(6-10)$ & $8(6-10)$ & $9(7-14)$ & $7(5-10)$ \\
\hline \multicolumn{5}{|l|}{ Hunt-Hess grade } \\
\hline 1 & $5(3.2)$ & $4(4.1)$ & $8(8.5)$ & $1(0.9)$ \\
\hline 2 & $7(4.5)$ & $3(3.1)$ & $7(7.4)$ & $1(0.9)$ \\
\hline 3 & $41(26.5)$ & $23(23.7)$ & $24(25.5)$ & $28(25.9)$ \\
\hline 4 & $64(41.3)$ & $44(45.4)$ & $41(43.6)$ & $41(38.0)$ \\
\hline 5 & $38(24.5)$ & $23(23.7)$ & $14(14.9)$ & $37(34.3)$ \\
\hline \multicolumn{5}{|l|}{ Modified Fisher scale } \\
\hline 1 & $19(12.3)$ & $6(6.2)$ & $7(7.4)$ & $12(11.1)$ \\
\hline 2 & $21(13.5)$ & $7(7.2)$ & $12(12.8)$ & $12(11.1)$ \\
\hline 3 & $63(40.6)$ & $40(41.2)$ & $38(40.4)$ & $42(38.9)$ \\
\hline 4 & $52(33.5)$ & $44(45.4)$ & $37(39.4)$ & $42(38.9)$ \\
\hline Hijdra scale & $18.3 \pm 7.4$ & $19.8 \pm 8.4$ & $18.8 \pm 7.7$ & $18.6 \pm 7.8$ \\
\hline Global cerebral oedema, initial CT & $41(26.5)$ & $32(33.0)$ & $26(27.7)$ & $28(25.9)$ \\
\hline Aneurysm size, mm & $8.7 \pm 6.7$ & $8.0 \pm 4.5$ & $7.5 \pm 3.9$ & $9.9 \pm 7.5$ \\
\hline APACHE II score & $17.5 \pm 7.0$ & $18.0 \pm 6.2$ & $16.2 \pm 6.5$ & $19.3 \pm 6.7$ \\
\hline \multicolumn{5}{|l|}{ Follow-up assessment } \\
\hline Rebleeding & $20(12.9)$ & $21(21.6)$ & $8(8.5)$ & $24(22.2)$ \\
\hline Global cerebral oedema, follow-up CT & $111(71.6)$ & $85(87.6)$ & $60(63.8)$ & $92(85.2)$ \\
\hline Hydrocephalus & $110(71.0)$ & $74(76.3)$ & $64(68.1)$ & $85(78.7)$ \\
\hline Intracranial pressure crisis & $95(61.3)$ & $75(77.3)$ & $61(64.9)$ & $74(68.5)$ \\
\hline Meningitis & $25(16.1)$ & $20(20.6)$ & $14(14.9)$ & $25(23.1)$ \\
\hline Pneumonia and/or pulmonary oedema & $96(61.9)$ & $73(75.3)$ & $55(58.5)$ & $84(77.8)$ \\
\hline Sepsis & $28(18.1)$ & $28(28.9)$ & $12(12.8)$ & $28(25.9)$ \\
\hline Exposure to hyperoxia & $28(18.1)$ & $36(37.1)$ & $18(19.1)$ & 32 (29.6) \\
\hline Exposure to hypocarbia & $25(16.1)$ & $23(23.7)$ & $15(16.0)$ & $21(19.4)$ \\
\hline
\end{tabular}

\section{Hyperoxia and baseline characteristics}

Among demographics, risk factors, underlying comorbidities and initial findings, there was no variable significantly related to hyperoxia (see online supplementary table S2).

\section{Hyperoxia and DCI}

Table 1 shows demographics, risk factors or comorbidities, findings on initial assessments, findings on follow-up assessments and an exposure to hyperoxia, assorted by DCI. DCI was observed in 97/252 (38.5\%) patients, and the median time interval from symptom onset to the occurrence of DCI was 6.0 days (mean \pm SD, 6.4 \pm 3.1 days; IQR, 4-8 days) (figures 2 and 3). Table 2 shows factors associated with DCI: highest grade of modified Fisher Scale $(p=0.012)$, global cerebral oedema on follow-up CT $(p=0.004)$, intracranial pressure crisis $(p=0.009)$, pneumonia and/or pulmonary oedema $(p=0.030)$, sepsis $(\mathrm{p}=0.046)$, and hyperoxia $(\mathrm{p}=0.001)$. Rebleeding was marginally associated with DCI $(p=0.070)$. Increase in oxygen burden was related to high proportion of patients with DCI (figure 4). In multiple logistic regression analysis, modified Fisher Scale grade $3(p=0.040)$ and grade $4(p=0.005)$, global cerebral oedema on follow-up CT $(p=0.004)$, pneumonia and/or pulmonary oedema $(p=0.010)$ and hyperoxia $(p<0.001)$ were independently associated with DCI.

\section{Hyperoxia and poor outcome}

mRS At 3 months was available in $202(80.2 \%)$ patients (see online supplementary table S3). Poor outcome occurred in 108/ $202(53.5 \%)$ patients. Table 3 shows the factors associated with poor outcome: older age $(p<0.001)$, APACHE II score $(\mathrm{p}=0.001)$, higher Hunt-Hess grade $(\mathrm{p}<0.001)$, aneurysm size $(p=0.006)$, rebleeding $(p=0.011)$, global cerebral oedema on follow-up CT $(p=0.001)$, pneumonia and/or pulmonary 

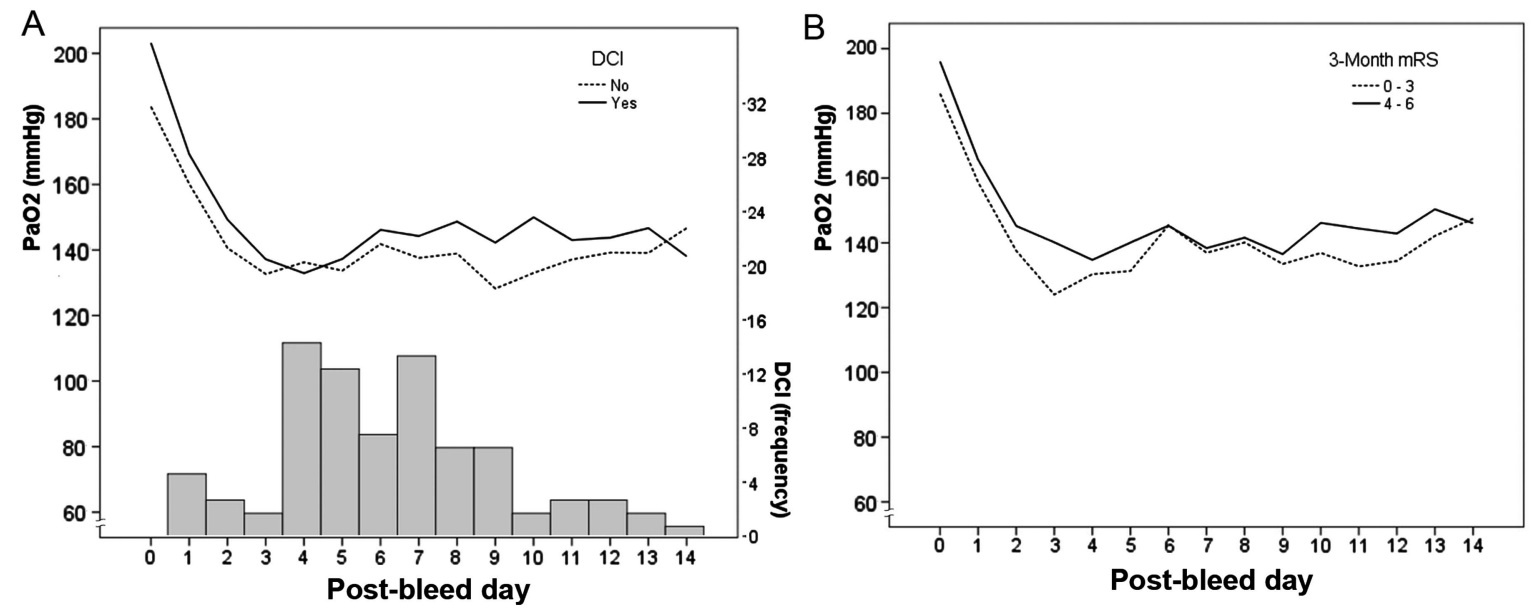

Figure 2 Average daily $\mathrm{PaO}_{2}$ on each postbleed day. $\mathrm{PaO}_{2}$ according to the development of (A) delayed cerebral ischaemia (DCI) and (B) poor outcome. Histogram shows the number of patients who developed $\mathrm{DCl}$.

oedema $(p=0.004)$, and sepsis $(p=0.022)$. Exposure to hyperoxia was marginally associated with poor outcome $(\mathrm{p}=0.087)$. Increase in oxygen burden was related to high proportion of patients with poor outcome (figure 4). In multiple logistic regression analysis, older age $(p=0.001)$, higher Hunt-Hess grade $(p=0.012)$, aneurysm size $(p=0.024)$, rebleeding $(p=0.035)$ and hyperoxia $(p=0.042)$ independently predicted poor outcome.

\section{DISCUSSION}

Our main finding is that exposure to hyperoxia was associated with an increased risk for DCI and poor outcome after SAH. As expected, given our selection of high-grade SAH patients, we had a high rate of DCI (38.5\%) and poor functional outcome $(53.5 \%)$. Exposure to hyperoxia was related to approximately three times the risk of DCI and twice the risk of poor 3-months outcome. This relationship was independent of the SAH severity and other comorbidities.

Currently, no guidelines for oxygen therapy in SAH exist. In this study, during the first week after $\mathrm{SAH}$, three quarters of patients were exposed to an average $\mathrm{PaO}_{2} \geq 123 \mathrm{~mm} \mathrm{Hg}$, and a quarter of patients were exposed to an average $\mathrm{PaO}_{2} \geq 173 \mathrm{~mm} \mathrm{Hg}$. As seen in our cohort, a recent study has shown that the risk of being exposed to hyperoxia is common in critically ill patients, and such condition was not addressed with any adjustments in ventilator settings. ${ }^{17}$

Hyperoxia causes oxidative stress by generation of reactive oxygen species and is known to cause lung injury. ${ }^{8} 18-20$ Detrimental cerebral effects of hyperoxia have been reported. Oxidative stress may cause brain injury through unavailability of nitric oxide, inflammation and neuronal necrosis. ${ }^{21} 22$ Hyperoxia decreases the availability of nitric oxide through reduced basal production, oxidative quenching and decreased release from the S-nitrosohemoglobin binding. ${ }^{21}$ This contributes to vasoconstriction and decrease in cerebral blood flow. ${ }^{10} 23-25$ Additionally, mitochondria-derived reactive oxygen species can cause neuronal inflammation. ${ }^{26} 27$ Hyperoxic reperfusion after global cerebral ischaemia has been reported to promote neuronal inflammation and long-term neuronal loss. ${ }^{22}$ At high pressure, hyperoxia has caused paralysis due to neuronal necrosis in rats. ${ }^{28}$ Furthermore, oxidative stress has a role in the formation and rupture of cerebral aneurysm through inflammation, activation of matrix metalloproteinases, and lipid peroxidation. ${ }^{29}$

Recently, hyperoxia has been shown to be associated with poor outcomes in other acute brain injury populations. A large
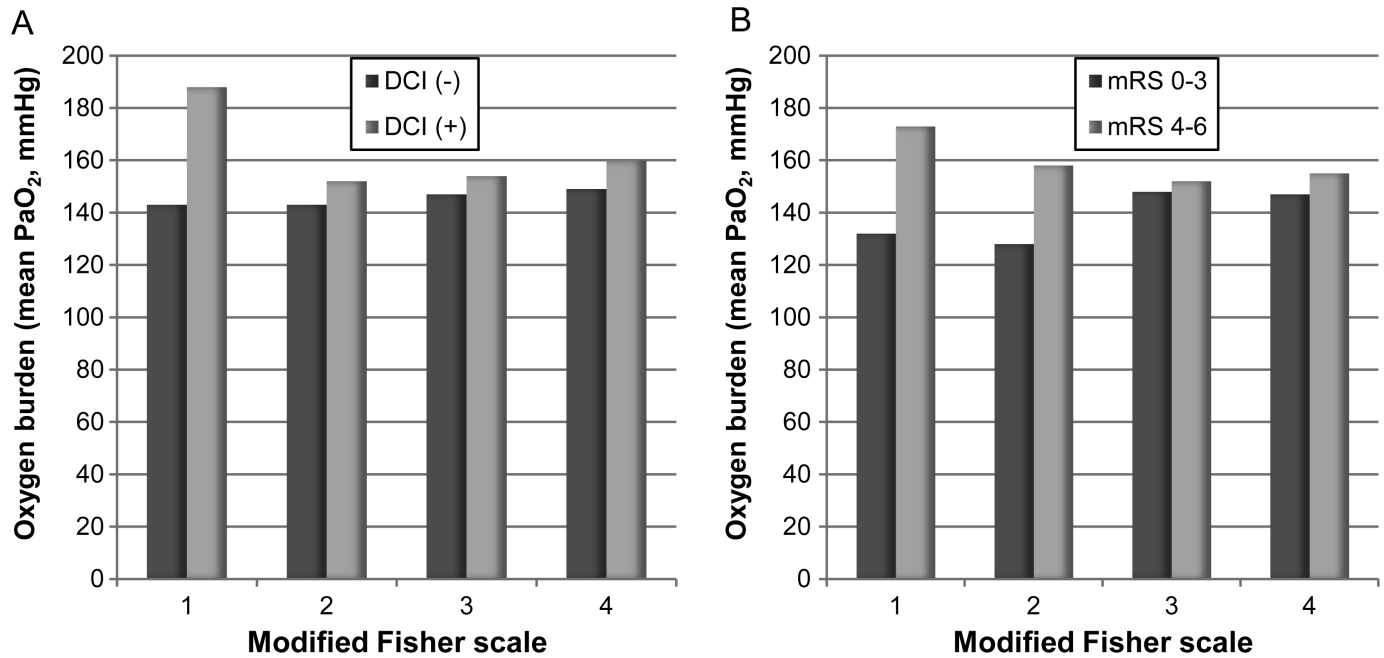

Figure 3 Oxygen burden according to severity of SAH. (A) In all grades of modified Fisher scale, oxygen burden was higher in patients with delayed cerebral ischaemia (DCI) than in those without $\mathrm{DCl}$. (B) Oxygen burden was higher in patients with poor outcome than in those without poor outcome. 
Table 2 Factors associated with the delayed cerebral ischaemia

\begin{tabular}{lll}
\hline & $\begin{array}{l}\text { Univariable } \\
\text { analysis } \\
\text { OR }(95 \% \mathrm{Cl})\end{array}$ & $\begin{array}{l}\text { Multivariable } \\
\text { analysis } \\
\text { OR }(95 \% \mathrm{Cl})\end{array}$ \\
\hline $\begin{array}{l}\text { Modified Fisher scale } \\
1-2\end{array}$ & $\begin{array}{l}\text { Reference } \\
1.95(0.93 \text { to } 4.10)\end{array}$ & $\begin{array}{l}\text { Reference } \\
2.29(1.04 \text { to } 5.04)\end{array}$ \\
3 & $2.60(1.24$ to 5.48$)$ & $3.15(1.42$ to 6.98$)$ \\
$\quad \begin{array}{l}\text { Rebleeding } \\
\text { Global cerebral oedema, }\end{array}$ & $2.87(0.95$ to 3.66$)$ & \\
follow-up CT & 2.81 .40 to 5.64$)$ & $2.95(1.41$ to 6.18$)$ \\
Intracranial pressure crisis & $2.15(1.21$ to 3.83$)$ & \\
$\begin{array}{l}\text { Pneumonia and/or pulmonary } \\
\text { oedema }\end{array}$ & $1.87(1.06$ to 3.29$)$ & $2.27(1.22$ to 4.22$)$ \\
$\begin{array}{l}\text { Sepsis } \\
\text { Exposure to hyperoxia }\end{array}$ & $1.84(1.01$ to 3.36$)$ & \\
\hline
\end{tabular}

multicentre cohort study found that exposure to hyperoxia was common following resuscitation from cardiac arrest, and associated with increase in hospital mortality. ${ }^{2}$ Retrospective studies in patients with traumatic brain injury have also found an association between worse functional outcomes and higher mortality in patients exposed to hyperoxia. ${ }^{3}{ }^{30}$ In both studies, oxidative stress due to reactive oxygen species has been postulated as the pathomechanism of the aggravation of initial brain injury. ${ }^{22}$ Conversely, studies have found that hyperoxia may be of therapeutic benefit for patients with brain injury. A pilot study showed that high-flow oxygen therapy via facemask transiently improved neurological deficits and MRI abnormalities compared with room-air therapy in patients with acute ischaemic stroke. ${ }^{6}$
Table 3 Factors associated with poor outcome

\begin{tabular}{|c|c|c|}
\hline & $\begin{array}{l}\text { Univariable analysis } \\
\text { OR }(95 \% \mathrm{Cl})\end{array}$ & $\begin{array}{l}\text { Multivariable analysis } \\
\text { OR }(95 \% \mathrm{CI})\end{array}$ \\
\hline Age, years & 1.04 (1.02 to 1.06$)$ & 1.05 (1.02 to 1.08$)$ \\
\hline Hunt-Hess grade & 1.78 (1.31 to 2.43$)$ & 1.74 (1.13 to 2.68$)$ \\
\hline Aneurysm size, mm & 1.08 (1.02 to 1.15$)$ & 1.08 (1.01 to 1.16$)$ \\
\hline APACHE II score & 1.07 (1.03 to 1.12$)$ & \\
\hline Rebleeding & 3.07 (1.31 to 7.22$)$ & 3.06 (1.09 to 8.64$)$ \\
\hline $\begin{array}{l}\text { Global cerebral oedema, } \\
\text { follow-up CT }\end{array}$ & 3.26 (1.66 to 6.42$)$ & \\
\hline $\begin{array}{l}\text { Pneumonia and/or } \\
\text { pulmonary oedema }\end{array}$ & 2.48 (1.35 to 4.58$)$ & \\
\hline Sepsis & 2.39 (1.14 to 5.03$)$ & \\
\hline Exposure to hyperoxia & 1.78 (0.92 to 3.44$)$ & 2.30 (1.03 to 5.12$)$ \\
\hline
\end{tabular}

A magnetic resonance spectroscopy study also found that normobaric oxygen therapy decreased lactate level and preserved $\mathrm{N}$-acetyl-aspartate peak in patients with acute ischaemic stroke. Other studies showed that hyperoxia was related to improvement of cerebral metabolism and reduction of intracranial pressure in patients with traumatic brain injury. ${ }^{4} 5$ At present, the role of hyperoxia remains controversial in patients with acute brain injury. Future clinical trials are warranted to address this issue.

DCI is an important complication after $\mathrm{SAH}$, increasing the mortality and morbidity for survivors. ${ }^{16} 31$ Complex pathophysiology is related to the development of DCI, including vasospasm, neurovascular inflammation and microcirculatory dysfunction. ${ }^{32}$ Narrowing of the lumen of large or small vessels
Figure 4 Relationship of oxygen doses to delayed cerebral ischaemia (DCl) and poor outcome. The proportion of patients with $\mathrm{DCl}$ and poor outcome increased in high oxygen burden.

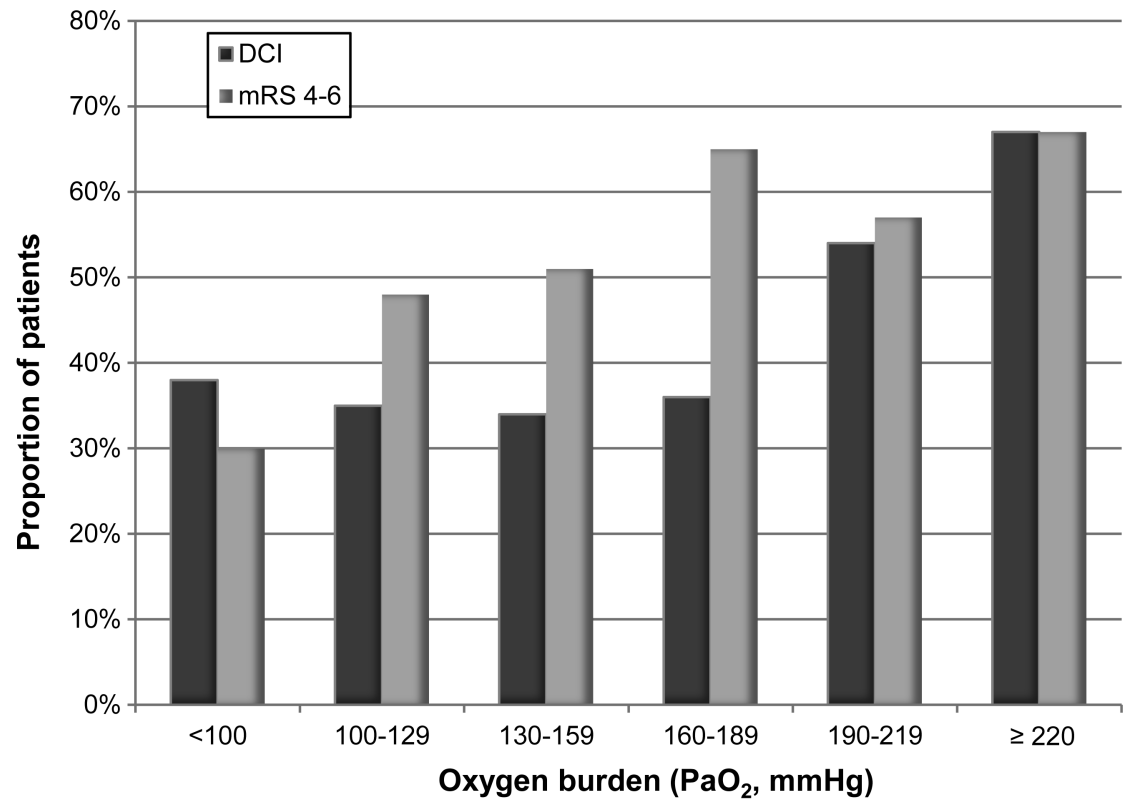

No. of patients

$\begin{array}{llllccr}\text { DCl (-) } & 8 & 44 & 55 & 32 & 12 & 4 \\ \text { DCl (+) } & 5 & 24 & 28 & 18 & 14 & 8 \\ \text { mRS 0-3 } & 7 & 29 & 33 & 13 & 9 & 3 \\ \text { mRS 4-6 } & 3 & 27 & 35 & 25 & 12 & 6\end{array}$


may cause ischaemia distal to the involved sites. Effects of hyperoxia on the vasoconstriction and reduction in cerebral blood flow may contribute to DCI. ${ }^{10}{ }^{23}$ Oxygen-free radicals may also be involved in neurovascular inflammation through activation of microglia and astrocytes. ${ }^{22} 2627$ Furthermore, hyperoxia can impair cerebral microcirculation by disturbing fibrinolysis and activation of the coagulation system. ${ }^{33}$ Vasoconstriction, inflammation and impaired cerebral microcirculation have all been postulated to be important processes for the development of DCI.

Our study has limitations. First, this was an observational study, and findings should be interpreted cautiously. Despite our findings of hyperoxia's association with DCI, hyperoxia may be a surrogate marker rather than a cause in the development of DCI. In a retrospective study, potential interactions among variables may not be completely excluded by multivariate analysis. Second, we chose to include only patients who were intubated and had $\mathrm{PaO}_{2}$ examined. Patients included in our study were older and had more severe symptoms than those who were excluded. This is a potential source of selection bias and it is unclear if our findings can be generalised to the low-grade $\mathrm{SAH}$ patient. Third, due to the design, we were also not able to obtain detailed information on ventilator settings, such as modes and fraction of inspired oxygen, shunting and metabolic status. However, regardless of the ventilator settings, we believe that the $\mathrm{PaO}_{2}$ levels were the most critical values regarding the development of DCI because the effect of ventilator settings, shunting and metabolic status would most likely work through $\mathrm{PaO}_{2}$ levels. Additionally, measurements of $\mathrm{PaO}_{2}$ were inconsistent across patients in terms of time points and interval. These limitations, due to retrospective nature, should be addressed in future prospective studies. Fourth, a consensus on the definition of hyperoxia in SAH is not available in terms of a level and duration of $\mathrm{PaO}_{2}$. A recently published large multicentre cohort study defined hyperoxia as $\mathrm{PaO}_{2}$ of $300 \mathrm{~mm} \mathrm{Hg}$ or greater in the first (within $24 \mathrm{~h}$ after admission) arterial blood-gas analysis. $^{2}$ However, their definition of hyperoxia was based on 'consensus of all authors', because the precise $\mathrm{PaO}_{2}$ level associated with maximal risk is unknown. ${ }^{2}$ Moreover, the previous study's population was cardiac arrest victims, whose secondary brain injury would occur mostly within $24 \mathrm{~h}$. On the contrary, in patients with $\mathrm{SAH}$, secondary brain injury in the form of DCI occurs typically between 3 and 14 days after SAH. ${ }^{16}$ Another study of patients with traumatic brain injury averaged $\mathrm{PaO}_{2}$ level during the first $24 \mathrm{~h}$ of hospital admission and defined hyperoxia as $\mathrm{PaO}_{2}$ levels of $200 \mathrm{~mm} \mathrm{Hg}$ or greater. ${ }^{3}$ This definition of hyperoxia, however, was arbitrarily based on the authors' clinical experiences of rarely seeing $\mathrm{PaO}_{2}$ values greater than $200 \mathrm{~mm} \mathrm{Hg}$. Moreover, to our best knowledge, there are no laboratory data to define hyperoxia in SAH population. Thus, we defined hyperoxia as the highest quartile of area under the curve of $\mathrm{PaO}_{2}$ based on our patients' data. Although hyperoxic $\mathrm{PaO}_{2}$ levels $(\geq 173 \mathrm{~mm} \mathrm{Hg}$ ) of our patients were moderate compared with $\mathrm{PaO}_{2}$ levels $(\geq 300$ and $\geq 200 \mathrm{~mm} \mathrm{Hg}$ ) of the previous studies, we believe that continuous exposure to excess oxygen could also be harmful. When our patients were exposed to higher oxygen doses, the risks of developing DCI and poor outcome further increased. We favour avoiding hyperoxia, but a cutoff value of $173 \mathrm{~mm} \mathrm{Hg}$ based on our retrospective study cannot be recommended. Further studies remain to be published to address the cutoff value.

In conclusion, our study suggests that exposure to hyperoxia may be associated with DCI and poor 3-month outcome in SAH patients requiring mechanical ventilation. Exposure to excess oxygen after SAH may represent a modifiable factor for morbidity and mortality in this population. Further studies are necessary to validate our findings and explore its clinical implications.

\section{Author affiliations}

${ }^{1}$ Department of Neurology, Asan Medical Center, University of Ulsan College of Medicine, Seoul, Republic of Korea

${ }^{2}$ Departments of Neurology and Neurosurgery, The University of Texas Medical School at Houston, Houston, Texas, USA

${ }^{3}$ Department of Neurology, Columbia University College of Physicians and Surgeons, New York, New York, USA

${ }^{4}$ Department of Neurology, University of Maryland School of Medicine, Baltimore, Maryland, USA

${ }^{5}$ Departments of Neurosurgery, Columbia University College of Physicians and Surgeons, New York, New York, USA

Acknowledgements We are grateful to Younsuk Lee, MD, PhD, Institute of Statistical Research and Department of Anesthesiology, Dongguk University College of Medicine, Goyang, Gyeonggi-do, Republic of Korea, for statistical assistance.

Contributors $\mathrm{S}-\mathrm{BJ}, \mathrm{HAC}$ and $\mathrm{KL}$ contributed to the article by participating in the concept and design. S-BJ, HAC, NB, JMS, HL, JC, ESC, SAM and KL: acquisition of data. S-BJ, HAC, NB and KL: analysis and interpretation of data. S-BJ, HAC and KL: drafting of the manuscript. S-BJ, HAC, NB, JMS, HL, JC, ESC, SAM and KL: critical revision of the manuscript for intellectual content.

\section{Competing interests None.}

\section{Patient consent Obtained.}

Ethics approval The institutional review board of New York Presbyterian Hospital. Provenance and peer review Not commissioned; externally peer reviewed.

\section{REFERENCES}

1 Pancioli AM, Broderick JP. Prehospital and emergency department care of the patient with acute stroke. In: Mohr JP, Choi DW, Grotta JC, Weir B, Wolf PA, eds. Stroke: pathophysiology, diagnosis, and management. 4th edn. Philadelphia, PA: Churchill Livinstones, 2004:905-18.

2 Kilgannon $\mathrm{JH}$, Jones $\mathrm{AE}$, Shapiro $\mathrm{NI}$, et al. Association between arterial hyperoxia following resuscitation from cardiac arrest and in-hospital mortality. JAMA 2010;303:2165-71.

3 Brenner M, Stein D, Hu P, et al. Association between early hyperoxia and worse outcomes after traumatic brain injury. Arch Surg 2012;147:1042-6.

4 Tolias CM, Reinert M, Seiler R, et al. Normobaric hyperoxia-induced improvement in cerebral metabolism and reduction in intracranial pressure in patients with severe head injury: a prospective historical cohort-matched study. J Neurosurgery 2004; 101:435-44.

5 Menzel M, Doppenberg EM, Zauner A, et al. Increased inspired oxygen concentration as a factor in improved brain tissue oxygenation and tissue lactate levels after severe human head injury. J Neurosurgery 1999;91:1-10.

6 Singhal $A B$, Benner $T$, Roccatagliata $L$, et al. A pilot study of normobaric oxygen therapy in acute ischemic stroke. Stroke 2005;36:797-802.

7 Singhal $A B$, Ratai $E$, Benner $T$, et al. Magnetic resonance spectroscopy study of oxygen therapy in ischemic stroke. Stroke 2007;38:2851-4.

8 Buonocore G, Perrone S, Tataranno ML. Oxygen toxicity: chemistry and biology of reactive oxygen species. Semin Fetal Neonatal Med 2010;15:186-90.

9 Becker LB. New concepts in reactive oxygen species and cardiovascular reperfusion physiology. Cardiovasc Res 2004;61:461-70.

10 Floyd TF, Clark JM, Gelfand R, et al. Independent cerebral vasoconstrictive effects of hyperoxia and accompanying arterial hypocapnia at 1 ATA. J Appl Physiol 2003:95:2453-61

11 Wartenberg KE, Schmidt JM, Claassen J, et al. Impact of medical complications on outcome after subarachnoid hemorrhage. Crit Care Med 2006;34:617-23.

12 Ko SB, Choi HA, Parikh G, et al. Multimodality monitoring for cerebral perfusion pressure optimization in comatose patients with intracerebral hemorrhage. Stroke 2011:42:3087-92.

13 Claassen J, Bernardini GL, Kreiter K, et al. Effect of cisternal and ventricular blood on risk of delayed cerebral ischemia after subarachnoid hemorrhage: the Fisher scale revisited. Stroke 2001;32:2012-20.

14 Claassen J, Carhuapoma JR, Kreiter KT, et al. Global cerebral edema after subarachnoid hemorrhage: frequency, predictors, and impact on outcome. Stroke 2002:33:1225-32.

15 Lord AS, Fernandez L, Schmidt JM, et al. Effect of rebleeding on the course and incidence of vasospasm after subarachnoid hemorrhage. Neurology 2012;78:31-7.

16 Frontera JA, Fernandez A, Schmidt JM, et al. Defining vasospasm after subarachnoid hemorrhage: what is the most clinically relevant definition? Stroke 2009:40:1963-8. 
17 de Graaff AE, Dongelmans DA, Binnekade JM, et al. Clinicians' response to hyperoxia in ventilated patients in a Dutch ICU depends on the level of FiO2. Intensive Care Med 2011;37:46-51.

18 Jackson RM. Pulmonary oxygen toxicity. Chest 1985;88:900-5.

19 Quinlan GJ, Lamb NJ, Tilley R, et al. Plasma hypoxanthine levels in ARDS: implications for oxidative stress, morbidity, and mortality. Am J Respir Crit Care Med 1997:155:479-84.

20 Griffith DE, Garcia JG, James HL, et al. Hyperoxic exposure in humans. Effects of 50 percent oxygen on alveolar macrophage leukotriene B4 synthesis. Chest 1992:101:392-7.

21 Calzia E, Asfar P, Hauser B, et al. Hyperoxia may be beneficial. Crit Care Med 2010;38:S559-68.

22 Hazelton JL, Balan I, Elmer Gl, et al. Hyperoxic reperfusion after global cerebral ischemia promotes inflammation and long-term hippocampal neuronal death. J Neurotrauma 2010;27:753-62.

23 Watson NA, Beards SC, Altaf N, et al. The effect of hyperoxia on cerebral blood flow: a study in healthy volunteers using magnetic resonance phase-contrast angiography. Eur J Anaesthesiol 2000;17:152-9.

24 Rousseau A, Bak Z, Janerot-Sjoberg B, et al. Acute hyperoxaemia-induced effects on regional blood flow, oxygen consumption and central circulation in man. Acta Physiol Scand 2005;183:231-40.
25 Singhal AB. Oxygen therapy in stroke: past, present, and future. Int I Stroke 2006;1:191-200.

26 Robertson CL, Scafidi S, McKenna MC, et al. Mitochondrial mechanisms of cell death and neuroprotection in pediatric ischemic and traumatic brain injury. Exp Neurol 2009;218:371-80.

27 Nakanishi H, Wu Z. Microglia-aging: roles of microglial lysosome- and mitochondriaderived reactive oxygen species in brain aging. Behav Brain Res 2009;201:1-7.

28 Balentine JD. Pathologic effects of exposure to high oxygen tensions. A review. N Engl J Med 1966;275:1038-40.

29 Starke RM, Chalouhi N, Ali MS, et al. The role of oxidative stress in cerebral aneurysm formation and rupture. Curr Neurovasc Res 2013;10:247-5.

30 Davis DP, Meade W, Sise MJ, et al. Both hypoxemia and extreme hyperoxemia may be detrimental in patients with severe traumatic brain injury. $J$ Neurotrauma 2009:26:2217-23.

31 Kassell NF, Peerless SJ, Durward QJ, et al. Treatment of ischemic deficits from vasospasm with intravascular volume expansion and induced arterial hypertension. Neurosurgery 1982;11:337-43.

32 Leng LZ, Fink ME, ladecola C. Spreading depolarization: a possible new culprit in the delayed cerebral ischemia of subarachnoid hemorrhage. Arch Neurol 2011;68:31-6.

33 Barazzone C, Belin D, Piguet PF, et al. Plasminogen activator inhibitor-1 in acute hyperoxic mouse lung injury. J Clin Invest 1996;98:2666-73. 